\title{
Crescimento e produtividade da mamoneira adubada com macronutrientes e micronutrientes
}

\author{
Liv Soares Severino(1), Gilvan Barbosa Ferreira( ${ }^{(1)}$, Cássia Regina de Almeida Moraes ${ }^{(1)}$, \\ Tarcísio Marcos de Souza Gondim ${ }^{(1)}$, Whertas Saldanha de Almeida Freire ${ }^{(2)}$, Diego Almeida de Castro(2), \\ Gleibson Dionízio Cardoso ${ }^{(1)}$ e Napoleão Esberard de Macedo Beltrão(1)
}

(1)Embrapa Algodão, Rua Oswaldo Cruz, no 1143, CEP 58107-720 Campina Grande, PB. E-mail: liv@cnpa.embrapa.br, gilvanbf@cnpa.embrapa.br, tarcisio@cnpa.embrapa.br, gleibson@cnpa.embrapa.br, nbeltrao@cnpa.embrapa.br, cramorae@ig.com.br (2)Consórcio Cenp Energia, Av. Sen. Virgílio Távora, no 1701, Sala 1306, CEP 60170-251 Fortaleza, CE.

Resumo - Um experimento foi implantado no Município de Quixeramobim, CE, com plantas de mamona (Ricinus communis L.), cv. BRS Nordestina, para verificar o efeito da adubação mineral com macronutrientes e micronutrientes no crescimento, na expressão sexual e na produtividade. Utilizou-se delineamento em blocos ao acaso, com três repetições e 14 tratamentos, em distribuição de matriz baconiana, com as doses de referência: $50 \mathrm{~kg} \mathrm{ha}^{-1}$ de N, $60 \mathrm{~kg} \mathrm{ha}^{-1}$ de P e $40 \mathrm{~kg} \mathrm{ha}^{-1}$ de K. Foram colhidos dados do crescimento, expressão sexual, produtividade e teor de óleo. As características ligadas ao crescimento foram pouco influenciadas pelas doses de nutrientes, mas detectou-se diferença no tratamento sem adubação em relação aos demais. O aumento nas doses de P promoveu a elevação do teor de óleo nas sementes. O aumento da disponibilidade de $\mathrm{N}$ e K promoveu alteração na expressão sexual da mamoneira, favorecendo o aumento de produtividade. A produtividade aumentou significativamente de 1.072 para $2.298 \mathrm{~kg} \mathrm{ha}^{-1}$ com o fornecimento de adubo nitrogenado.

Termos para indexação: Ricinus communis, expressão sexual, teor de óleo.

\section{Growth and yield of castor bean fertilized with macronutrients and micronutrients}

\begin{abstract}
A trial was carried out at Quixeramobim, CE, with castor bean plants (Ricinus communis L.), cv. BRS Nordestina, in order to evaluate the effect of mineral fertilization with macronutrients and micronutrients on growth, sexual expression and yield. A randomized blocks design with three replications and 14 treatments originated from a baconian matrix was adopted. Reference doses were $50 \mathrm{~kg} \mathrm{ha}^{-1}$ of N, $60 \mathrm{~kg} \mathrm{ha}^{-1}$ of P and $40 \mathrm{~kg} \mathrm{ha}^{-1}$ of K. Data on growth, sexual expression, yield and oil content were measured. Characteristics related to plant growth were low affected by treatments except in the absence of fertilization. Seed oil content increased in response to $\mathrm{P}$ doses. Higher availability of $\mathrm{N}$ and $\mathrm{K}$ promoted some alteration in the sexual expression favorable for increasing yield. Yield increased from 1,072 to 2,298 $\mathrm{kg} \mathrm{ha}^{-1}$ due to $\mathrm{N}$ fertilizer.
\end{abstract}

Index terms: Ricinus communis, sexual expression, oil content.

\section{Introdução}

A adubação é uma das principais tecnologias usadas para aumentar a produtividade e a rentabilidade das culturas, embora tenha alto custo e possa aumentar o risco do investimento agrícola. Contudo, há carência de informações sobre a tecnologia para fertilização do solo na cultura da mamoneira. Além disso, existem poucos relatos sobre o comportamento da mamoneira sob diferentes condições, como cultivares, níveis de fertilidade do solo, clima e disponibilidade de água. Essa planta é sensível à acidez do solo e exigente em fertilidade, sendo possível aumentar sua produtividade pelo adequado fornecimento de nutrientes por meio da adubação (Souza \& Neptune, 1976; Weiss, 1983).

A mamoneira exporta da área de cultivo cerca de $80 \mathrm{~kg} \mathrm{ha}^{-1}$ de $\mathrm{N}, 18 \mathrm{~kg} \mathrm{ha}^{-1}$ de $\mathrm{P}_{2} \mathrm{O}_{5}, 32 \mathrm{~kg} \mathrm{ha}^{-1}$ de $\mathrm{K}_{2} \mathrm{O}$, $13 \mathrm{~kg} \mathrm{ha}^{-1}$ de $\mathrm{CaO}$ e $10 \mathrm{~kg} \mathrm{ha}^{-1}$ de $\mathrm{MgO}$ para cada $2.000 \mathrm{~kg} \mathrm{ha}^{-1}$ de baga produzida. No entanto, a quantidade de nutriente absorvida aos 133 dias da germinação chega a 156, 12, 206, 19 e $21 \mathrm{~kg} \mathrm{ha}^{-1}$ de $\mathrm{N}, \mathrm{P}_{2} \mathrm{O}_{5}, \mathrm{~K}_{2} \mathrm{O}, \mathrm{CaO}$ e $\mathrm{MgO}$, respectivamente Cannecchio Filho \& Freire, 1958; 
Nakagawa \& Neptune, 1971), o que indica alto requerimento de nutrientes para obtenção de produtividade adequada.

Os teores foliares de $\mathrm{N}$ chegam a $41,3 \mathrm{~g} \mathrm{~kg}^{-1}$ aos 64 dias da germinação e é comum se encontrar na torta de mamona 45 a $46 \mathrm{~g} \mathrm{~kg}^{-1}$ desse nutriente, teor considerado muito alto (Nakagawa \& Neptune, 1971; Raij et al., 1996).

Este trabalho teve o objetivo de avaliar os efeitos da adubação mineral com N, P, K, Ca, Mg e micronutrientes (B, Cu, $\mathrm{Zn}, \mathrm{Mn}$ e $\mathrm{Fe}$ ) sobre o crescimento, expressão sexual, produtividade e teor de óleo nas sementes da cultivar de mamona BRS Nordestina, nas condições edafoclimáticas do Município de Quixeramobim, CE.

\section{Material e Métodos}

Sementes da cultivar BRS Nordestina foram plantadas na Fazenda Normal, Município de Quixeramobim, CE, em fevereiro de 2004. Utilizou-se delineamento em blocos ao acaso, com três repetições e 14 tratamentos, distribuídos em matriz baconiana (Tabela 1), na qual um dos nutrientes é fornecido em quantidades variáveis, enquanto os outros são mantidos em um nível referencial de 50, 60 e $40 \mathrm{~kg} \mathrm{ha}^{-1}$ de $\mathrm{N}, \mathrm{P}$ e K, respectivamente. Testou-se também a adição de $330 \mathrm{~kg} \mathrm{ha}^{-1}$ de calcário dolomítico (como fonte de Ca e Mg) e uma mistura de micronutrientes contendo o equivalente a $1 \mathrm{~kg} \mathrm{ha}^{-1}$ de B, 0,5 kg ha-1 de Cu, $1 \mathrm{~kg} \mathrm{ha}^{-1}$ de Fe, $1 \mathrm{~kg} \mathrm{ha}^{-1}$ de Mg e $1 \mathrm{~kg} \mathrm{ha}^{-1}$ de Zn.

Cada parcela constou de sete linhas com $6 \mathrm{~m}$ de comprimento, espaçadas entre si de 2,5 m e $1 \mathrm{~m}$ entre plantas. Foram consideradas úteis apenas as cinco linhas centrais, totalizando $105 \mathrm{~m}^{2}$ de área total e $75 \mathrm{~m}^{2}$ de área útil.

O N nas doses de 50 e $100 \mathrm{~kg} \mathrm{ha}^{-1}$ foi aplicado 50\% no plantio e $50 \%$ depois de 45 dias. Os demais nutrientes, e o $\mathrm{N}$ na dose de $25 \mathrm{~kg} \mathrm{ha}^{-1}$, foram aplicados somente no plantio. Como fonte de nutrientes, utilizou-se sulfato de amônio, superfosfato triplo e cloreto de potássio. O calcário utilizado continha $32 \%$ de $\mathrm{CaO}$ e $12 \%$ de $\mathrm{MgO}$, com PRNT de $45 \%$. Os micronutrientes foram todos fornecidos na forma de sulfato. Os fertilizantes foram colocados na cova de plantio imediatamente antes do semeio.

Plantaram-se três sementes por cova, realizando-se desbastes para uma planta por cova aos 15 dias depois da emergência. Durante o ciclo da cultura, realizou-se controle das plantas daninhas com enxada e controle químico de formigas. Não ocorreram doenças ou pragas que necessitassem de controle químico.

O solo possuía baixa fertilidade, apresentando acidez leve $(\mathrm{pH} 5,6)$ e presença de $\mathrm{Al}$ em teor baixo $\left(1,0 \mathrm{mmol}_{\mathrm{C}} \mathrm{dm}^{-3}\right)$; a saturação de bases apresentava-se relativamente baixa (57\%), baixo teor de P (2,15 mg $\left.\mathrm{dm}^{-3}\right)$ e baixo teor de matéria orgânica $\left(11,48 \mathrm{~g} \mathrm{~kg}^{-1}\right)$.

A estação chuvosa de 2004 foi atípica, com precipitações acima da média histórica. Entre janeiro e agosto, foram registrados $1.270 \mathrm{~mm}$ de chuva, sendo que em janeiro e metade de fevereiro, antes do plantio, choveu $511 \mathrm{~mm}$. Depois do plantio, as chuvas totalizaram $758 \mathrm{~mm}$, sendo distribuídas em $108 \mathrm{~mm}$ em fevereiro, $264 \mathrm{~mm}$ em março, $64 \mathrm{~mm}$ em abril, $89 \mathrm{~mm}$ em maio, $186 \mathrm{~mm}$ em junho, $42 \mathrm{~mm}$ em julho e $5 \mathrm{~mm}$ em agosto. A quantidade e distribuição da chuva foi considerada satisfatória para o bom desenvolvimento da cultura.

Tabela 1. Distribuição das doses de N, P, K e micronutrientes em matriz baconiana.

\begin{tabular}{cccccc}
\hline Tratamento & $\mathrm{N}\left(\mathrm{kg} \mathrm{ha}^{-1}\right)$ & $\mathrm{P}\left(\mathrm{kg} \mathrm{ha}^{-1}\right)$ & $\mathrm{K}\left(\mathrm{kg} \mathrm{ha}^{-1}\right)$ & Micronutrientes $^{(1)}$ & ${\text { Calcário }\left(\mathrm{kg} \mathrm{ha}^{-1}\right)}$ \\
\hline 1 & 0 & 0 & 0 & 0 & 0 \\
2 & 50 & 60 & 40 & $1 \mathrm{x}$ & 330 \\
3 & 0 & 60 & 40 & $1 \mathrm{x}$ & 330 \\
4 & 25 & 60 & 40 & $1 \mathrm{x}$ & 330 \\
5 & 100 & 60 & 40 & $1 \mathrm{x}$ & 330 \\
6 & 50 & 0 & 40 & $1 \mathrm{x}$ & 330 \\
7 & 50 & 120 & 40 & $1 \mathrm{x}$ & 330 \\
8 & 50 & 60 & 0 & $1 \mathrm{x}$ & 330 \\
9 & 50 & 60 & 20 & $1 \mathrm{x}$ & 330 \\
10 & 50 & 60 & 80 & $2 \mathrm{x}$ & 330 \\
11 & 50 & 60 & 40 & $1 \mathrm{x}$ & 330 \\
12 & 50 & 60 & 40 & 40 & 0 \\
13 & 50 & 60 & & \\
\hline
\end{tabular}

${ }^{(1)} 1 \mathrm{x}$ : dose de micronutrientes equivalente a $1 \mathrm{~kg} \mathrm{ha}^{-1}$ de $\mathrm{B}, 0,5 \mathrm{~kg} \mathrm{ha}^{-1}$ de $\mathrm{Cu}, 1 \mathrm{~kg} \mathrm{ha}^{-1}$ de Fe, $1 \mathrm{~kg} \mathrm{ha}^{-1}$ de Mn e $1 \mathrm{~kg}$ ha ${ }^{-1}$ de Zn; $2 \mathrm{x}$ : dobro da dose $1 \mathrm{x}$. 
Aos 130 dias depois do plantio, foram tomadas as medidas de altura da planta, altura de inserção do primeiro cacho, diâmetro do caule na base, número de nós até o primeiro cacho, e comprimento da parte masculina e feminina do cacho. Calculou-se a razão entre o comprimento da parte feminina e masculina e o comprimento total do cacho. Aos 210 dias depois do plantio, os frutos foram colhidos e pesados, e estimou-se a massa das sementes multiplicando-se a massa dos frutos pelo fator de conversão 0,6144, proposto por Severino et al. (2005). Para medição do teor de óleo, amostras das sementes foram secadas em estufa a $70^{\circ} \mathrm{C}$ por 24 horas e submetidas à análise por ressonância nuclear magnética.

Os dados obtidos foram submetidos à análise de variância e análise de regressão por polinômios ortogonais, conforme Ferreira (1996). Nas características em que se encontrou significância, calcularam-se os coeficientes da regressão. Nos tratamentos de calcário, considerou-se que a detecção de efeito significativo pelo teste $\mathrm{F}$ da análise de variância corresponde à diferença significativa entre as médias, por haver somente duas médias.

\section{Resultados e Discussão}

Entre as características ligadas ao crescimento da planta - altura da planta, altura de inserção do primeiro cacho, número de nós até o primeiro cacho, comprimento médio do internódio e diâmetro caulinar os únicos efeitos significativos foram observados no tratamento testemunha sobre a altura do primeiro cacho, número, comprimento médio do internódio e diâmetro caulinar, indicando maior crescimento vegetativo nas plantas adubadas, conforme Nakagawa \& Neptune (1971).

Entre as características ligadas à expressão sexual da mamoneira, vários efeitos significativos foram observados (Tabela 2). O comprimento do cacho sofreu efeito linear das doses de $\mathrm{N}$ e efeito significativo na testemunha. $\mathrm{O}$ comprimento da parte feminina aumentou de forma quadrática com as doses de $\mathrm{N}$ e sofreu efeito significativo na comparação entre os tratamentos com adubação e sem adubação. O comprimento da parte masculina do cacho foi influenciado significativamente pelas doses de $\mathrm{N}$ (efeito linear), $\mathrm{P}$ (efeito linear), K (efeito quadrático) e pela adição de calcário. O teor de óleo apresentou baixo coeficiente de variação (3,2\%), sendo observado efeito linear significativo das doses de P. Quanto à produtividade, houve alto coeficiente de variação (23,9\%) e os únicos efeitos significativos foram observados nas doses de $\mathrm{N}$ (efeito quadrático) e na comparação dos tratamentos com adubação e sem adubação.

A expressão sexual da mamoneira é influenciada por diversos fatores, entre eles a idade da planta e do racemo, duração do dia, temperatura e poda (Popova \& Moshkin, 1986; Beltrão et al., 2001). A produtividade está diretamente ligada à proporção entre comprimento das partes feminina e masculina do cacho, sendo que essas características são influenciadas pela disponibilidade de nutrientes no solo.

Devido à grande necessidade de $\mathrm{N}$ - para otimizar os processos bioquímicos na planta, especialmente a fotossíntese, e suprir o forte dreno de proteína das sementes (Marschner, 1995) -, aos baixos teores de matéria orgânica $\left(11,5 \mathrm{~g} \mathrm{dm}^{-3}\right)$ e de $\mathrm{P}\left(2,2 \mathrm{mg} \mathrm{dm}^{-3}\right)$ e aos teores médios de $\mathrm{K}$ disponíveis (102 $\mathrm{mg} \mathrm{dm}^{-3}$ ) encontrados no solo, a resposta em produtividade refletiu a fertilidade encontrada, sendo mais intensa quando comparada com a ausência de adubação, na seguinte ordem de importância: $\mathrm{N}>\mathrm{P}>$ micronutrientes $>$ calcário $>\mathrm{K}$. A fertilidade natural do solo permitiu a expressão de apenas $46,6 \%$ do potencial de produção da cultura (Tabela 2). Os resultados obtidos concordam com os descritos por Nakagawa \& Neptune (1971) e demonstram a necessidade de adubação balanceada para otimizar a produtividade e a produção de óleo dessa cultura.

A altura das plantas foi muito influenciada pelos tratamentos. Nas plantas que não receberam adubação, a altura foi 31\% menor, reduzindo-se de 2,62 m para $1,8 \mathrm{~m}$ (Tabela 3), valor que foi significativo a 6,6\% pelo teste F. Nas demais doses dos nutrientes avaliados, as plantas cresceram muito, mas houve pequena diferença entre os tratamentos. Segundo Weiss (1983), sabe-se que variedades de qualquer espécie vegetal podem responder de forma diferente em crescimento e produção a determinado nível de nutrição, de forma que plantas selecionadas para crescer em determinado nível de nutriente foram adaptadas para produzir o máximo naquele nível. É por isso que raramente a mamoneira é cultivada em solos muito férteis, pois nessas condições ela tende a produzir grande massa vegetativa em detrimento da produção de sementes.

A cultivar BRS Nordestina é um exemplo de planta que tem tendência a excessivo crescimento vegetativo quando há disponibilidade de nutrientes e umidade no solo, condições observadas neste experimento. 
Em alguns casos, como na dose 2x de micronutrientes, as plantas atingiram a média de $3,13 \mathrm{~m}$. Este crescimento vegetativo excessivo, além de dificultar a colheita, diminui a produtividade, pois o gasto de energia para formação de folhas e caules compete com a produção das sementes. Esse fato deve ser observado na condução de plantios irrigados, onde se deve evitar o fornecimento de água em abundância e fertilizantes de forma desequilibrada, sob risco de causar crescimento excessivo em altura e diminuição na produtividade. Essa realidade é comum para a maioria das plantas de mamoneira de portes médio e alto (Azevedo et al., 2001), e impõe desafio adicional ao melhoramento de plantas: sintetizar variedades com maior índice de colheita, que ao mesmo tempo tenha como características distintivas menor crescimento arbustivo, maior produtividade de baga e de óleo e uso eficiente de adubos e corretivo.
A altura do primeiro cacho é uma característica ligada à precocidade da planta, sendo considerada mais precoce a planta que lança o primeiro cacho em menor altura. Porém, neste experimento, a altura do cacho foi maior nas plantas adubadas não por causa de precocidade, mas pelo comprimento médio do internódio que aumentou de 4,5 para $6 \mathrm{~cm}$ (Tabela 3), não sendo percebida diferença no número de nós até o aparecimento do primeiro cacho.

Devido ao excessivo crescimento vegetativo, o diâmetro do caule das plantas foi grande, com média de $40 \mathrm{~mm}$. Apenas nas plantas que não receberam a adubação, o diâmetro foi significativamente menor: 32,6 mm em comparação com as plantas adubadas que tiveram diâmetro de 43,5 mm.

Tabela 2. Médias do comprimento do cacho, comprimento da parte masculina, comprimento da parte feminina, razão entre comprimento da parte feminina e masculina, teor de óleo, produtividade, ganho em relação à testemunha e perda de potencial produtivo da mamoneira nos tratamentos com e sem adubação em função de doses de N, P, K, micronutrientes e calcário (Ca + Mg).

\begin{tabular}{|c|c|c|c|c|c|c|c|c|}
\hline \multirow[t]{2}{*}{ Tratamentos $^{(1)}$} & \multicolumn{4}{|c|}{ Comprimento } & \multirow{3}{*}{$\begin{array}{c}\text { Teor de óleo } \\
(\%)\end{array}$} & \multirow{3}{*}{$\begin{array}{l}\text { Produtividade } \\
\qquad\left(\mathrm{kg} \mathrm{ha}^{-1}\right)\end{array}$} & \multirow{2}{*}{$\begin{array}{l}\text { Ganho em } \\
\text { relação à } \\
\text { testemunha }\end{array}$} & \multirow{2}{*}{$\begin{array}{c}\text { Perda de } \\
\text { potencial } \\
\text { produtivo }\end{array}$} \\
\hline & Cacho & $\begin{array}{l}\text { Parte } \\
\text { fem. }\end{array}$ & $\begin{array}{l}\text { Parte } \\
\text { masc. }\end{array}$ & $\begin{array}{c}\text { Razão } \\
\text { fem./masc. }\end{array}$ & & & & \\
\hline \multicolumn{7}{|l|}{ Adubação } & & \\
\hline Sem & $38,8^{* *}$ & 9,9 & $29,0 * *$ & 3,0 & 48,1 & $1.072,0 * *$ & 0 & 55 \\
\hline Com & $49,4 * *$ & 10,9 & $38,5 * *$ & 3,6 & 48,1 & $2.298,9 * *$ & 114 & 4 \\
\hline \multicolumn{9}{|c|}{ Nitrogênio $\left(\mathrm{kg} \mathrm{ha}^{-1}\right)$} \\
\hline 0 & $41,0^{\mathrm{L}}$ & $12,5^{\mathrm{L}}$ & $28,5^{\mathrm{Q}}$ & $2,3^{Q}$ & 49,9 & $1.476,5^{\mathrm{Q}}$ & 38 & 38 \\
\hline 25 & $51,6^{\mathrm{L}}$ & $14,7^{\mathrm{L}}$ & $36,9^{\mathrm{Q}}$ & $2,6^{\mathrm{Q}}$ & 49,6 & $2.217,1^{\mathrm{Q}}$ & 107 & 8 \\
\hline 50 & $49,4^{\mathrm{L}}$ & $10,9^{\mathrm{L}}$ & $38,5^{\mathrm{Q}}$ & $3,6^{\mathrm{Q}}$ & 48,1 & $2.298,9^{Q}$ & 114 & 4 \\
\hline 100 & $51,0^{\mathrm{L}}$ & $14,8^{\mathrm{L}}$ & $36,2^{\mathrm{Q}}$ & $2,5^{\mathrm{Q}}$ & 49,4 & $1.972,3^{Q}$ & 84 & 18 \\
\hline \multicolumn{9}{|c|}{ Fósforo $\left(\mathrm{kg} \mathrm{ha}^{-1}\right)$} \\
\hline 0 & 47,1 & $11,7^{\mathrm{L}}$ & 35,4 & 3,0 & $47,6^{\mathrm{L}}$ & $1.494,1$ & 39 & 38 \\
\hline 30 & 51,0 & $14,9^{\mathrm{L}}$ & 36,0 & 2,5 & $48,8^{\mathrm{L}}$ & $1.728,0$ & 61 & 28 \\
\hline 60 & 49,4 & $10,9^{\mathrm{L}}$ & 38,5 & 3,6 & $48,1^{\mathrm{L}}$ & $2.298,9$ & 114 & 4 \\
\hline 120 & 52,2 & $13,3^{\mathrm{L}}$ & 38,9 & 3,0 & $50,2^{\mathrm{L}}$ & $2.171,7$ & 103 & 9 \\
\hline \multicolumn{9}{|c|}{ Potássio $\left(\mathrm{kg} \mathrm{ha}^{-1}\right)$} \\
\hline 0 & 53,5 & $14,3^{\mathrm{Q}}$ & 39,2 & $2,8^{\mathrm{Q}}$ & 49,6 & $1.864,8$ & 74 & 22 \\
\hline 30 & 54,3 & $11,9^{\mathrm{Q}}$ & 42,4 & $3,6^{\mathrm{Q}}$ & 49,4 & $1.898,1$ & 77 & 21 \\
\hline 60 & 49,4 & $10,9^{Q}$ & 38,5 & $3,6^{\mathrm{Q}}$ & 48,1 & $2.298,9$ & 114 & 4 \\
\hline 120 & 47,9 & $13,0^{\mathrm{Q}}$ & 34,9 & $2,7^{Q}$ & 49,6 & $1.720,5$ & 60 & 28 \\
\hline \multicolumn{9}{|c|}{ Calcário $\left(\mathrm{kg} \mathrm{ha}^{-1}\right)$} \\
\hline 0 & 55,7 & $13,9 *$ & 41,8 & 3,0 & 49,2 & $1.881,1$ & 75 & 22 \\
\hline 330 & 49,4 & $10,9 *$ & 38,5 & 3,6 & 48,1 & $2.298,9$ & 114 & 4 \\
\hline \multicolumn{9}{|l|}{ Micronutrientes } \\
\hline 0 & 54,5 & 11,7 & 42,8 & 3,8 & 48,4 & $1.736,0$ & 62 & 28 \\
\hline $1 \mathrm{x}$ & 49,4 & 10,9 & 38,5 & 3,6 & 48,1 & $2.298,9$ & 114 & 4 \\
\hline $2 x$ & 49,6 & 12,3 & 37,3 & 3,0 & 47,2 & $2.062,9$ & 92 & 14 \\
\hline
\end{tabular}

${ }^{(1)}$ Todos os tratamentos receberam a adubação padrão de $50 \mathrm{~kg} \mathrm{ha}^{-1}$ de $\mathrm{N}, 60 \mathrm{~kg} \mathrm{ha}^{-1}$ de P, $40 \mathrm{~kg} \mathrm{ha}^{-1}$ de $\mathrm{K}, 330 \mathrm{~kg}$ ha-1 de calcário e micronutrientes, havendo apenas a variação que caracterizava o tratamento. ${ }^{(2)}$ Os ganhos de produção em relação à testemunha são calculados sobre a produtividade obtida pela testemunha absoluta e equivale a: Ganho $=\{[100 \mathrm{x}$ (produtividade do tratamento avaliado/produtividade da testemunha) $]$ - 100 $\}$. ${ }^{(3)}$ A perda de produtividade é calculada sobre a máxima produtividade estimada (2.399 $\mathrm{kg} \mathrm{ha}^{-1} \mathrm{com}$ a dose teórica de $58,6 \mathrm{~kg}^{-1}$ de $\mathrm{N}$ ), usando a fórmula: Perda de produção potencial $=\{100$ - [(produtividade do tratamento avaliado/2.399) x 100] $\}$. * e **Significativo a 5 e $1 \%$ de probabilidade pelo teste $\mathrm{F}$, respectivamente. ${ }^{\mathrm{L}}$ e QEfeito linear e quadrático, respectivamente, na análise de regressão por polinômios ortogonais. 
Observou-se consistente aumento do teor de óleo nas sementes em resposta ao aumento nas doses de P. Entre a dose zero e $100 \mathrm{~kg} \mathrm{ha}^{-1}$ de $\mathrm{P}$, o teor aumentou de 47,6 para 50,2\%. A equação de regressão do teor de óleo em função das doses de $\mathrm{P}\left(\mathrm{kg} \mathrm{ha}^{-1}\right)$ foi: teor de óleo $(\%)=0,0234 \mathrm{P}+47,68$. O P participa de importantes reações químicas da fisiologia da planta, com destaque para os processos ligados ao fluxo de energia, compondo a molécula de ATP e outras moléculas de grande importância, e entre os produtos comumente armazenados em sementes (óleos, proteínas e carboidratos), os ácidos graxos componentes dos óleos são os que mais exigem gastos de ATP por grama de produto armazenado (Beltrão et al., 2001). É possível que o maior suprimento deste nutriente seja uma prática viável para obtenção de maior teor de óleo na cultura da mamona, no entanto, esse efeito precisa ser confirmado em outros ambientes e genótipos.

A produtividade foi influenciada pela adubação. Nas plantas que receberam a adubação de referência, a produtividade foi $114 \%$ maior que nas plantas que não foram adubadas. Devido ao alto coeficiente de variação (23,9\%), detectou-se efeito significativo apenas das doses de $\mathrm{N}$ (efeito quadrático). A equação de regressão da produtividade em razão de doses de $\mathrm{N}\left(\mathrm{kg} \mathrm{ha}^{-1}\right)$ foi: produtividade $\left(\mathrm{kg} \mathrm{ha}^{-1}\right)=-0,257 \mathrm{~N}^{2}+30,1 \mathrm{~N}+1517$. Por essa equação, estima-se a produtividade máxima equivalente a $2.399 \mathrm{~kg} \mathrm{ha}^{-1} \mathrm{com}$ a dose de $58,6 \mathrm{~kg} \mathrm{ha}^{-1}$ de N. Porém, percebe-se tendência a aumento da produtividade também nas doses de $\mathrm{P}, \mathrm{K}$, calcário e micronutrientes. $\mathrm{O}$ fornecimento em excesso de qualquer dos nutrientes prejudicou a produtividade, principalmente porque houve boa disponibilidade de água e a cultivar tem forte tendência a crescimento em altura.

A adubação de referência (50-60-40 N- $\mathrm{P}_{2} \mathrm{O}_{5}-\mathrm{K}_{2} \mathrm{O}$ + calagem + micronutrientes) promoveu um aumento de $114 \%$ na produtividade comparada com a parcela não adubada (Tabela 2). O único nutriente que pode ser

Tabela 3. Médias de altura da planta, altura do primeiro cacho, número de nós até o 1o cacho, comprimento médio do internódio e diâmetro caulinar da mamoneira nos tratamentos com adubação e sem adubação e em função de doses de N, P, K, micronutrientes e calcário ( $\mathrm{Ca}+\mathrm{Mg})$.

\begin{tabular}{|c|c|c|c|c|c|}
\hline Tratamentos $^{(1)}$ & Altura da planta & Altura do $1^{0}$ cacho & $\mathrm{N}^{0}$ de nós até $1^{\underline{0}}$ cacho & $\begin{array}{r}\text { Comprimento do } \\
\text { internódio }(\mathrm{cm})\end{array}$ & $\begin{array}{l}\text { Diâmetro caulinar } \\
(\mathrm{mm})\end{array}$ \\
\hline \multicolumn{6}{|l|}{ Adubação } \\
\hline Sem & 1,8 & $0,9 *$ & 20,9 & $4,5^{*}$ & $32,6 *$ \\
\hline Com & 2,6 & $1,3 *$ & 21,7 & $6,0^{*}$ & $43,5 *$ \\
\hline \multicolumn{6}{|c|}{ Nitrogênio $\left(\mathrm{kg} \mathrm{ha}^{-1}\right)$} \\
\hline 0 & 2,0 & 1,0 & 21,0 & 4,9 & 35,2 \\
\hline 25 & 2,6 & 1,3 & 21,5 & 5,9 & 41,6 \\
\hline 50 & 2,6 & 1,3 & 21,7 & 6,0 & 43,5 \\
\hline 100 & 2,3 & 1,2 & 22,7 & 5,3 & 36,9 \\
\hline \multicolumn{6}{|c|}{ Fósforo $\left(\mathrm{kg} \mathrm{ha}^{-1}\right)$} \\
\hline 0 & 2,4 & 1,2 & 21,7 & 5,4 & 38,9 \\
\hline 30 & 2,4 & 1,1 & 22,0 & 5,1 & 38,1 \\
\hline 60 & 2,6 & 1,3 & 21,7 & 6,0 & 43,5 \\
\hline 120 & 2,4 & 1,3 & 22,1 & 6,1 & 42,1 \\
\hline \multicolumn{6}{|c|}{ Potássio $\left(\mathrm{kg} \mathrm{ha}^{-1}\right)$} \\
\hline 0 & 2,4 & 1,3 & 23,3 & 5,3 & 39,1 \\
\hline 30 & 2,8 & 1,3 & 21,9 & 5,9 & 45,1 \\
\hline 60 & 2,6 & 1,3 & 21,7 & 6,0 & 43,5 \\
\hline 120 & 2,2 & 1,1 & 22,4 & 5,0 & 37,3 \\
\hline \multicolumn{6}{|c|}{ Calcário (kg ha-1) } \\
\hline 0 & 3,1 & 1,4 & 22,1 & 6,4 & 44,0 \\
\hline 330 & 2,6 & 1,3 & 21,7 & 6,0 & 43,5 \\
\hline \multicolumn{6}{|l|}{ Micronutrientes } \\
\hline 0 & 3,0 & 1,5 & 22,9 & 6,6 & 44,4 \\
\hline $1 x$ & 2,6 & 1,3 & 21,7 & 6,0 & 43,5 \\
\hline $2 x$ & 3,1 & 1,3 & 22,0 & 5,8 & 45,7 \\
\hline
\end{tabular}

(1)Todos os tratamentos receberam a adubação padrão de $50 \mathrm{~kg} \mathrm{ha}^{-1}$ de $\mathrm{N}, 60 \mathrm{~kg} \mathrm{ha}^{-1}$ de P, $40 \mathrm{~kg} \mathrm{ha}^{-1}$ de $\mathrm{K}, 330 \mathrm{~kg}$ ha ${ }^{-1}$ de calcário e micronutrientes, havendo apenas a variação que caracterizava o tratamento. * Significativo a $5 \%$ de probabilidade pelo teste $\mathrm{F}$. 
ajustado para melhorar a produtividade é o N. Com o uso de 58,6 $\mathrm{kg} \mathrm{ha}^{-1}$ desse nutriente, a produtividade alcançada foi cerca de $124 \%$ maior que a da testemunha absoluta, dando um ganho líquido de $10 \%$ sobre a recomendação padrão utilizada. Quanto aos demais nutrientes, o uso de doses menores ou maiores promove perda de produtividade e de investimento do recurso aplicado.

\section{Conclusões}

1. A adubação da mamoneira, cultivar BRS Nordestina, com fertilizantes químicos, promove considerável aumento da produtividade.

2. O teor de óleo nas sementes aumenta consistentemente em resposta às doses de $\mathrm{P}$, mas não é influenciado pelos demais nutrientes.

3. A adubação mineral com $\mathrm{N}$ e K promove mudança na expressão sexual da mamoneira, favorecendo o aumento de produtividade.

\section{Agradecimentos}

Ao Consórcio Cenp Energia e ao Fundo de Desenvolvimento Científico e Tecnológico (Fundeci) do Banco do Nordeste, pelo apoio financeiro; ao CNPq, pela concessão de bolsa de pesquisa à Dra. Cássia Moraes; à Ematerce, pelo apoio logístico.

\section{Referências}

AZEVEDO, D.M.P. de; NÓBREGA, L.B. da; LIMA, E.F.; BATISTA, F.A.S.; BELTRÃO, N.E. de M. Manejo cultural. In: AZEVEDO, D.M.P. de; LIMA, E.F. (Ed.). O agronegócio da mamona no Brasil. Brasília: Embrapa Informação Tecnológica, 2001. p.121-160.

BELTRÃO, N.E. de M.; SILVA, L.C.; VASCONCELOS, O.L.; AZEVEDO, D.M.P. de; VIEIRA, D.J. Fitologia. In: AZEVEDO, D.M.P. de; LIMA, E.F. (Ed.). O agronegócio da mamona no Brasil. Brasília: Embrapa Informação Tecnológica, 2001. p.37-61.

CANECCHIO FILHO, V.; FREIRE, E.S. Adubação da mamoneira: experiências preliminares. Bragantia, v.17, p.243-259, 1958.

FERREIRA, P.V. Estatística experimental aplicada à agronomia. 2.ed. Maceió: Edufal, 1996. 606p.

MARSCHNER, H. Mineral nutrition of higher plants. $2^{\text {nd }}$ ed. London: Academic, 1995. 889p.

NAKAGAWA, J.; NEPTUNE, A.M.L. Marcha de absorção de nitrogênio, fósforo, potássio, cálcio e magnésio na cultura da mamoneira (Ricinus communis L.) cultivar Campinas. Anais da Escola Superior de Agricultura Luiz de Queiroz, v.28, p.323337, 1971.

POPOVA, G.M.; MOSHKIN, V.A. Flower biology. In: MOSHKIN, V.A. Castor. New Delhi: Amerind, 1986. p.11-64.

RAIJ, B. van; CANTARELLA, H.; QUAGGIO, J.A.; FURLANI, A.M.C. (Ed.). Recomendação de adubação e calagem para o Estado de São Paulo. 2.ed. Campinas: Instituto Agronômico, 1996. 285p. (Boletim técnico, 100).

SEVERINO, L.S.; MORAES, C.R.A.; GONDIM, T.M.S.; CARDOSO, G.D.; SANTOS, J.W. Fatores de conversão do peso de cachos e frutos para peso de sementes de mamona. Campina Grande: Embrapa Algodão, 2005. 14p. (Boletim de pesquisa, 56).

SOUZA, E.A.; NEPTUNE, A.M.L. Resposta da cultura de Ricinus communis L. à adubação e calagem. Científica, v.4, p.274-281, 1976. WEISS, E.A. Oilseed crops. London: Longman, 1983. 659p.

Recebido em 26 de janeiro de 2005 e aprovado em 19 de julho de 2005 